\title{
SUPPLEMENTARY MATERIAL \\ GaGa: a Parsimonious and Flexible Model for Differential Expression Analysis
}

\author{
David Rossell \\ Biostatistics and Bioinformatics Unit \\ Institute for Research in Biomedicine of Barcelona \\ 08028 Barcelona, Spain \\ rosselldavid@gmail.com
}

\section{Contents}

1 Model fitting schemes 2

$1.1 \quad$ EM algorithm $\ldots \ldots \ldots \ldots \ldots \ldots \ldots$

1.2 Fully Bayesian fit . . . . . . . . . . . . . . . . . . . . 4

1.3 Gamma approximation to Gamma Shape distribution . . . . . 5

$\begin{array}{lll}2 & \text { Bayesian FDR control } & 6\end{array}$

2.1 Bayesian procedure for gene differential expression analysis . . 6

\begin{tabular}{lll}
\hline 2.2 & Frequentist operating characteristics of the Bayesian procedure & 7
\end{tabular}

$2.3 \quad$ Frequentist FDR for the Armstrong dataset $\ldots \ldots \ldots 9$

3 Goodness of fit in the Armstrong dataset 10

4 Gene ontology analysis for MAQC study 13 
Here we detail procedures to fit the GaGa and MiGaGa models, to obtain lists of differentially expressed genes and assess their quality, and to evaluate model goodness-of-fit. We detail the MAQC data gene ontology analysis.

Section 1 describes several model fitting schemes and inspects the quality of the gamma approximation to the gamma shape distribution, which is heavily used by these schemes. Section 2 describes a procedure for Bayesian FDR control and discusses how to assess its frequentist FDR. In Section 3 we illustrate how to assess model goodness-of-fit. Section 4 provides some detail regarding the MAQC data gene ontology analysis discussed in the paper's main body.

\section{Model fitting schemes}

Section 1.1 describes the Expectation-Maximization (EM) algorithm that we used to fit the GaGa and MiGaGa models in the paper. Section 1.2 provides a fully Bayesian alternative scheme to fit the model. The computational efficiency of the EM and fully Bayesian schemes depends critically on the use of approximations to the Gamma Shape distribution. In Section 1.3 we inspect the approximation quality in two examples.

\subsection{EM algorithm}

Compared to the fully Bayesian fit described in Section 1.2 , the EM algorithm is a computationally efficient procedure to fit the model as it avoids posterior sampling. We formulate the MiGaGa model by introducing latent variables $c_{i}$ that specify the mixture component that each gene belongs to

$$
c_{i} \sim \operatorname{Mult}(1, \boldsymbol{\rho}), \text { indep. for } i=1, \ldots, n,
$$

where $\boldsymbol{\rho}=\left(\rho_{1}, \ldots, \rho_{M}\right)$ are the mixture weights and $M$ is the number of mixture components. The GaGa model corresponds to the particular case $M=1$.

The EM algorithm requires evaluating the likelihood $f(\mathbf{x} \mid \boldsymbol{\omega})$ of the hyperparameters $\boldsymbol{\omega}=\left(\boldsymbol{\rho}, \boldsymbol{\pi}, \boldsymbol{\alpha}_{\mathbf{0}}, \boldsymbol{\nu}, \beta, \mu\right)$. Due to independence conditional on $\boldsymbol{\omega}$ we can evaluate the likelihood as the product of the gene-wise likelihoods, that is

$$
f(\mathbf{x} \mid \boldsymbol{\omega})=\prod_{i=1}^{n} \sum_{c_{i}=1}^{m} \sum_{\delta_{i}=0}^{H-1} f\left(\mathbf{x}_{i} \mid c_{i}, \delta_{i}, \boldsymbol{\omega}\right) \pi_{\delta_{i}} \rho_{c_{i}}
$$


where $f\left(\mathbf{x}_{i} \mid c_{i}, \delta_{i}, \boldsymbol{\omega}\right)=$

$$
\left[\frac{\left(\alpha_{0 c_{i}} / \nu_{c_{i}}\right)^{\alpha_{0 c_{i}}}(\beta / \mu)^{\beta}}{\Gamma\left(\alpha_{0 c_{i}}\right) \Gamma(\beta)}\right]^{N_{\delta_{i}}} \prod_{k=1}^{N_{\delta_{i}}} \frac{1}{C\left(\boldsymbol{J}_{i, \delta_{i}}, \beta, \beta / \mu-P_{i}, \alpha_{0 c_{i}}, \alpha_{0 c_{i}} / \nu_{c_{i}}, \boldsymbol{S}_{i, \delta_{i}}\right)},
$$

and $C\left(\boldsymbol{J}_{i, \delta_{i}}, \beta, \beta / \mu-P_{i}, \alpha_{0 c_{i}}, \alpha_{0 c_{i}} / \nu_{c_{i}}, \boldsymbol{S}_{i, \delta_{i}}\right)$ is the Gamma Shape distribution normalization constant (see main paper, Section 4). Notice that the computational efficiency of the EM algorithm relies critically on evaluating this normalization constant quickly, which we are able to do with our Gamma approximation to the Gamma Shape distribution described in the main paper (Section 4).

The EM algorithm requires obtaining some initial guess for $\boldsymbol{\omega}=\hat{\boldsymbol{\omega}}^{(0)}$ and initializing $b=0$. Then the following two steps are repeated until convergence is achieved.

1. M-step: Set $b=b+1$. Maximize $f(\mathbf{x} \mid \boldsymbol{\omega})$ with respect to $\left(\boldsymbol{\alpha}_{\mathbf{0}}, \boldsymbol{\nu}, \beta, \mu\right)$, setting $\boldsymbol{\rho}=\hat{\boldsymbol{\rho}}^{(b-1)}, \boldsymbol{\pi}=\hat{\boldsymbol{\pi}}^{(b-1)}$. This requires the use of numerical optimization routines. Set $\left(\hat{\boldsymbol{\alpha}}_{0}, \hat{\boldsymbol{\nu}}, \hat{\beta}, \hat{\mu}\right)$ equal to the argmax.

2. E-step: estimate

$$
\hat{\pi}_{l}^{(b)}=\frac{1}{n} \sum_{i=1}^{n} P\left(\delta_{i}=l \mid \hat{\boldsymbol{\alpha}}_{0}, \hat{\boldsymbol{\nu}}, \hat{\beta}, \hat{\mu}, \mathbf{x}\right)
$$

for $l=0, \ldots, H-1$ and

$$
\hat{\rho}_{m}^{(b)}=\frac{1}{n} \sum_{i=1}^{n} \sum_{l=0}^{H-1} P\left(c_{i}=m \mid \delta_{i}=l, \hat{\boldsymbol{\alpha}}_{0}, \hat{\boldsymbol{\nu}}, \hat{\beta}, \hat{\mu}, \mathbf{x}\right) P\left(\delta_{i}=l \mid \hat{\boldsymbol{\alpha}}_{0}, \hat{\boldsymbol{\nu}}, \hat{\beta}, \hat{\mu}, \mathbf{x}\right),
$$

where

$$
\begin{aligned}
& P\left(\delta_{i}=l \mid \hat{\boldsymbol{\alpha}}_{0}, \hat{\boldsymbol{\nu}}, \hat{\beta}, \hat{\mu}, \mathbf{x}\right) \propto \sum_{m=1}^{M} f\left(\mathbf{x}_{i} \mid c_{i}=m, \delta_{i}=l, \hat{\boldsymbol{\alpha}}_{0}, \hat{\boldsymbol{\nu}}, \hat{\beta}, \hat{\mu}\right) \hat{\rho}_{m}^{(b-1)} \hat{\pi}_{l}^{(b-1)}, \\
& P\left(c_{i}=m \mid \delta_{i}=l, \hat{\boldsymbol{\alpha}}_{0}, \hat{\boldsymbol{\nu}}, \hat{\beta}, \hat{\mu}, \mathbf{x}\right) \propto f\left(\mathbf{x}_{i} \mid c_{i}=m, \delta_{i}=l, \hat{\boldsymbol{\alpha}}_{0}, \hat{\boldsymbol{\nu}}, \hat{\beta}, \hat{\mu}\right) \hat{\rho}_{m}^{(b-1)} \\
& \text { and } f\left(\mathbf{x}_{i} \mid c_{i}=m, \delta_{i}=l, \hat{\boldsymbol{\alpha}}_{0}, \hat{\boldsymbol{\nu}}, \hat{\beta}, \hat{\mu}\right) \text { is as in (3). }
\end{aligned}
$$




\subsection{Fully Bayesian fit}

Here we describe how to fit GaGa/MiGaGa models via MCMC posterior simulation. We complete the Bayesian model with hyper-priors:

$$
\begin{array}{r}
\alpha_{0 m} \sim \operatorname{Ga}\left(a_{\alpha_{0}}, b_{\alpha_{0}}\right) ; \nu_{m} \sim \operatorname{IGa}\left(a_{\nu}, b_{\nu}\right) \text { indep. for } m=1, \ldots, M \\
\beta \sim \operatorname{Ga}\left(a_{\beta}, b_{\beta}\right) ; \mu \sim \operatorname{IGa}\left(a_{\mu}, b_{\mu}\right) \\
\boldsymbol{\pi} \sim \operatorname{Dirichlet}(\mathbf{p}) \\
\boldsymbol{\rho} \sim \operatorname{Dirichlet}(\mathbf{r}) .
\end{array}
$$

Eliciting prior distributions can be natural in microarray studies, since there usually is some degree of prior knowledge. For example, the investigator may have an idea about what proportion of DE genes to expect. As another example, many normalization procedures result in values that are below 15 in the log-scale.

We consider two MCMC implementations, both of which are available in the Bioconductor package gaga: random-walk Metropolis-Hastings (4) and Gibbs sampling (3). The random-walk Metropolis-Hastings scheme allows sampling from the posterior distribution of the hyper-parameters $\boldsymbol{\omega}$ without the need to sample gene-specific parameters, and therefore each MCMC iteration is quicker than that of a Gibbs sampler. This strategy is straightforward to implement given the hyper-parameter likelihood $f(\mathbf{x} \mid \boldsymbol{\omega})$ in (2). In our implementation we use gamma proposals with expected values equal to the current hyper-parameter values.

In practice, we found the Gibbs sampler to have better mixing properties when the dimensionality of $\boldsymbol{\omega}$ increases, such as for the MiGaGa model. The Gibbs sampler requires two main steps.

- STEP 1: sample the gene-specific parameters $(\mathbf{c}, \boldsymbol{\delta}, \boldsymbol{\alpha}, \boldsymbol{\lambda})$ given the hyper-parameters $\boldsymbol{\omega}$ and the observed data $\mathbf{x}$. Given $\boldsymbol{\omega}$ and $\mathbf{x},\left(c_{i}, \delta_{i}, \alpha_{i}, \boldsymbol{\lambda}_{i}\right)$ are independent across $i=1, \ldots, n$. The expression pattern and mixture component indicators can be sampled according to the following probabilities:

$$
\begin{gathered}
P\left(\delta_{i}=l \mid \boldsymbol{\alpha}_{\mathbf{0}}, \boldsymbol{\nu}, \beta, \mu, \mathbf{x}\right) \propto \sum_{m=1}^{M} f\left(\mathbf{x}_{i} \mid c_{i}=m, \delta_{i}=l, \boldsymbol{\alpha}_{\mathbf{0}}, \boldsymbol{\nu}, \beta, \mu\right) \rho_{m} \pi_{l}, \\
P\left(c_{i}=m \mid \delta_{i}=l, \boldsymbol{\alpha}_{\mathbf{0}}, \boldsymbol{\nu}, \beta, \mu, \mathbf{x}\right) \propto f\left(\mathbf{x}_{i} \mid c_{i}=m, \delta_{i}=l, \boldsymbol{\alpha}_{\mathbf{0}}, \boldsymbol{\nu}, \beta, \mu\right) \rho_{m},
\end{gathered}
$$

where $f\left(\mathbf{x}_{i} \mid c_{i}=m, \delta_{i}=l, \boldsymbol{\alpha}_{\mathbf{0}}, \boldsymbol{\nu}, \beta, \mu\right)$ is as in (3). The Gamma parameters $\left(\alpha_{i}, \lambda_{i, k}\right)$ are sampled conditionally on $\left(\delta_{i}, c_{i}\right)$ as follows:

$$
\begin{aligned}
\alpha_{i} \mid c_{i}, \delta_{i}, \boldsymbol{\omega}, \mathbf{x} & \sim \operatorname{GaS}\left(\boldsymbol{J}_{i, \delta_{i}}, \beta, \beta / \mu-P_{i}, \alpha_{0 c_{i}}, \alpha_{0 c_{i}} / \nu_{c_{i}}, \boldsymbol{S}_{i, \delta_{i}}\right) \\
\lambda_{i, k} \mid \alpha_{i}, c_{i}, \delta_{i}, \boldsymbol{\omega}, \mathbf{x} & \sim \operatorname{IGa}\left(\alpha_{i} J_{i, \delta_{i}, k}+\alpha_{0 c_{i}}, \alpha_{0 c_{i}} / \nu_{c_{i}}+\alpha_{i} S_{i, \delta_{i}, k}\right),
\end{aligned}
$$


where GaS denotes the Gamma Shape distribution. Its definition and details on how to obtain random draws are provided in the main paper (Section 4).

- STEP 2: sample $\boldsymbol{\omega}$ given $(\mathbf{c}, \boldsymbol{\delta}, \boldsymbol{\alpha}, \boldsymbol{\lambda})$ and $\mathbf{x}$. The hyper-parameters $(\beta, \mu)$ are conditionally independent of $\left(\boldsymbol{\alpha}_{0}, \boldsymbol{\nu}\right)$ given $(\mathbf{c}, \boldsymbol{\delta}, \boldsymbol{\alpha}, \boldsymbol{\lambda})$ and $\mathbf{x}$, and $(\boldsymbol{\pi}, \boldsymbol{\rho})$ are also conditionally independent of $\left(\boldsymbol{\alpha}_{0}, \boldsymbol{\nu}, \beta, \mu\right)$. The hyper-parameters are sampled from the following distributions:

$$
\begin{aligned}
\alpha_{0 m} \mid \mathbf{c}, \boldsymbol{\delta}, \boldsymbol{\alpha}, \boldsymbol{\lambda}, \mathbf{x} & \sim \operatorname{GaS}\left(\sum_{i=1}^{n} I\left(c_{i}=m\right) N_{\delta_{i}}, a_{\alpha_{0}}, b_{\alpha_{0}}+S_{\lambda m}, a_{\nu}, b_{\nu}, S_{\lambda m}^{\prime}\right) \\
\nu_{m} \mid \alpha_{0 m}, \mathbf{c}, \boldsymbol{\delta}, \boldsymbol{\alpha}, \boldsymbol{\lambda}, \mathbf{x} & \sim \mathrm{IGa}\left(a_{\nu}+\alpha_{0 m} \sum_{i=1}^{n} I\left(c_{i}=m\right) N_{\delta_{i}}, b_{\nu}+\alpha_{0 m} S_{\lambda m}^{\prime}\right) \\
\beta \mid \mathbf{c}, \boldsymbol{\delta}, \boldsymbol{\alpha}, \boldsymbol{\lambda}, \mathbf{x} & \sim \operatorname{GaS}\left(\sum_{i=1}^{n} N_{\delta_{i}}, a_{\beta}, b_{\beta}-S_{\alpha}^{\prime}, a_{\mu}, b_{\mu}, S_{\alpha}\right) \\
\mu \mid \beta, \mathbf{c}, \boldsymbol{\delta}, \boldsymbol{\alpha}, \boldsymbol{\lambda}, \mathbf{x} & \sim \mathrm{IGa}\left(a_{\mu}+\beta \sum_{i=1}^{n} N_{\delta_{i}}, b_{\mu}+\beta S_{\alpha}\right)
\end{aligned}
$$

for $m=1, \ldots, M$. Here $S_{\lambda m}=\sum_{i=1}^{n} \sum_{k=1}^{N_{\delta_{i}}} I\left(c_{i}=m\right) \lambda_{i, k}, S_{\lambda m}^{\prime}=$ $\sum_{i=1}^{n} \sum_{k=1}^{N_{\delta_{i}}} I\left(c_{i}=m\right) 1 / \lambda_{i, k}, S_{\alpha}=\sum_{i=1}^{n} \sum_{k=1}^{N_{\delta_{i}}} \alpha_{i}$ and $S_{\alpha}^{\prime}=\sum_{i=1}^{n} \sum_{k=1}^{N_{\delta_{i}}} \log \left(\alpha_{i}\right)$.

The remaining hyper-parameters are sampled as follows:

$$
\begin{aligned}
& \boldsymbol{\pi} \mid \mathbf{c}, \boldsymbol{\delta}, \boldsymbol{\alpha}, \boldsymbol{\lambda}, \mathbf{x} \sim \operatorname{Dirichlet}\left(p_{1}+\sum_{i=1}^{n} \mathrm{I}\left(\delta_{i}=0\right), \ldots, p_{H-1}+\sum_{i=1}^{n} \mathrm{I}\left(\delta_{i}=H-1\right)\right) \\
& \boldsymbol{\rho} \mid \mathbf{c}, \boldsymbol{\delta}, \boldsymbol{\alpha}, \boldsymbol{\lambda}, \mathbf{x} \sim \operatorname{Dirichlet}\left(r_{1}+\sum_{i=1}^{n} \mathrm{I}\left(c_{i}=1\right), \ldots, r_{M}+\sum_{i=1}^{n} \mathrm{I}\left(c_{i}=M\right)\right)
\end{aligned}
$$

\subsection{Gamma approximation to Gamma Shape distribu- tion}

Figure 1 shows the gamma shape distribution and its gamma approximation for two randomly selected cases that were encountered in posterior simulations for the MiGaGa model. The density in panel (a) arises as the posterior of the shape parameter for a single gene with a sample size of 5 observations per group, whereas panel (b) results as the posterior of a parameter shared by a large number of genes i.e. it represents a situation with a large sample size. The approximation error is below $10^{-5}$ for the density and $10^{-14}$ for the normalization constant $C(\boldsymbol{a}, b, c, d, r, \boldsymbol{s})$. 
(a)

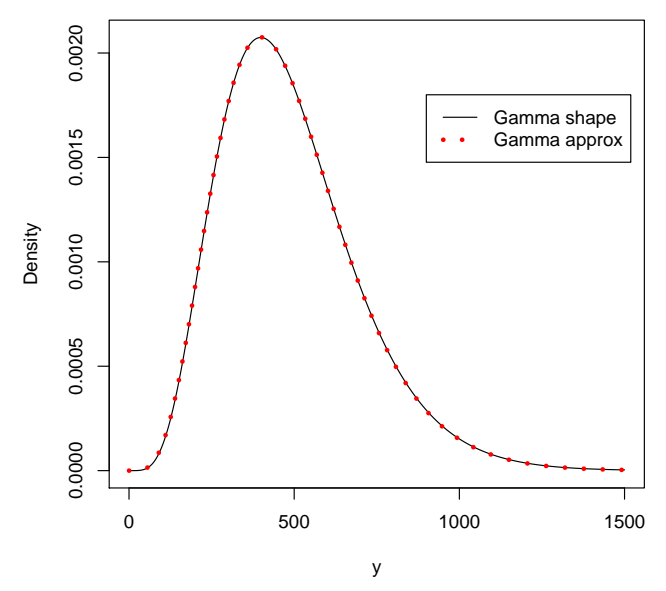

(b)

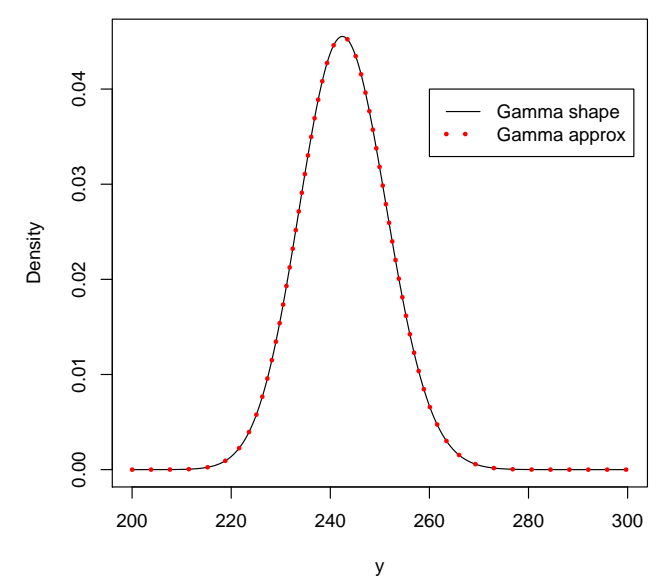

Figure 1: Gamma approximation to the gamma shape distribution. Parameter values are $(a): a=10 ; b=0.90 ; c=-18.71 ; d=1181.49 ; r=7907.76 ; s=65.02$ (b): $a=1532, b=.16, c=3469, d=.16, r=.016, s=159.5$

\section{Bayesian FDR control}

Section 2.1 describes a Bayesian procedure for differential expression that minimizes the Bayesian False Negative Rate while controlling the Bayesian False Discovery Rate below a user-specified threshold. In Section 2.2 we discuss a procedure to assess the frequentist operating characteristics of the Bayesian procedure, and in Section 2.3 we apply it to Armstrong's data (1).

\subsection{Bayesian procedure for gene differential expression analysis}

Here we detail the Bayesian procedure that minimizes the Bayesian FNR subject to the Bayesian FDR being below a threshold $\alpha$. Let $\delta_{1}, \ldots, \delta_{n}$ denote the expression pattern that each gene follows, e.g. if there are only two groups to be compared $\delta_{i}=0$ denotes the null hypothesis that both groups are equal and $\delta_{i}=1$ denotes that they are different. Denote as $d_{i}=d_{i}(\mathbf{x})$ the pattern that gene $i$ is assigned to, i.e. $d_{i}=0$ means that we declare the gene as equally expressed (EE) and $d_{i} \neq 0$ that we declare it differentially expressed (DE). The false negative (FNP) and false discovery proportions (FDP) can be written as: 


$$
\begin{aligned}
& \mathrm{FNP}=\frac{\sum_{i=1}^{n} \mathrm{I}\left(d_{i}=0\right) \mathrm{I}\left(\delta_{i} \neq 0\right)}{\sum_{i=1}^{n} \mathrm{I}\left(d_{i}=0\right)} \\
& \mathrm{FDP}=\frac{\sum_{i=1}^{n} \mathrm{I}\left(d_{i}=1\right) \mathrm{I}\left(\delta_{i}=0\right)}{\sum_{i=1}^{n} \mathrm{I}\left(d_{i}=1\right)}
\end{aligned}
$$

where $\mathrm{I}(\cdot)$ is the indicator function. That is, the FNP is the proportion of genes declared EE that are actually DE, and the FDP is the proportion of genes declared DE that are actually EE. The Bayesian FNR and Bayesian FDR are defined as the expected FNP and FDP, respectively, where the expectation is taken with respect to the posterior distribution of $\delta_{1}, \ldots, \delta_{n}$ (5). For any fixed decisions $d_{1}, \ldots, d_{n}$, one can evaluate the Bayesian FNR and FDR simply as

$$
\begin{aligned}
\mathrm{BFNR} & =\frac{\sum_{i=1}^{n} \mathrm{I}\left(d_{i}=0\right)\left(1-v_{i 0}\right)}{\sum_{i=1}^{n} \mathrm{I}\left(d_{i}=0\right)} \\
\mathrm{BFDR} & =\frac{\sum_{i=1}^{n} \mathrm{I}\left(d_{i}=1\right) v_{i 0}}{\sum_{i=1}^{n} \mathrm{I}\left(d_{i}=1\right)},
\end{aligned}
$$

where $v_{i 0}=P\left(\delta_{i}=0 \mid \mathbf{x}\right)$ is the posterior probability that gene $i$ is EE. (6) showed that, in a setup with only two hypotheses, the optimal rule to minimize BFNR subject to $\mathrm{BFDR}<\alpha$ is to declare as $\mathrm{DE}$ all genes with $v_{i 0}$ below a certain threshold $t$, i.e. $d_{i}=\mathrm{I}\left(v_{i 0}<t\right)$, where $t$ is the minimum value such that $\mathrm{BFDR} \leq \alpha$. Note that $\mathrm{BFNR}$ and $\mathrm{BFDR}$ only take into account whether a gene was classified into pattern 0 or not. Therefore, when minimizing BFNR subject to $\mathrm{BFDR} \leq \alpha$ it makes no difference whether a particular gene is assigned to pattern 1 or pattern 2, say. We propose the obvious: given that a gene is declared DE, we assign it to the pattern with highest posterior probability, i.e. $\delta_{i}=\mathrm{I}\left(v_{i 0}<t\right) \times \operatorname{argmax}_{k \in\{1, \ldots, H-1\}}\left(v_{i k}\right)$. It is straightforward to see that, for any fixed BFNR and BFDR, this rule maximizes the expected number of genes correctly classified into their expression pattern.

\subsection{Frequentist operating characteristics of the Bayesian procedure}

In this section we review the algorithm that (7) proposed to estimate the frequentist FDR for any given procedure, i.e. the expected FDP in (8) when the procedure is applied under repeated sampling. As a first step, one 
obtains bootstrap samples from the original data, in such a way that the sample mean and variance of each gene are roughly preserved and that it represents a sample under the complete null hypothesis that no genes are DE. Then one repeatedly applies the procedure to each bootstrap dataset, obtaining an estimate of the number of false positives, and compares that to the number of genes found in the original dataset. More specifically, the algorithm is as follows:

\section{Algorithm 1}

1. Apply the procedure to the original dataset, and denote the number of genes declared to be DE as $P$. Denote as $\bar{X}_{i}$ and $S_{i}$ the sample mean and standard deviation of the gene expression measurements for gene $i=1 \ldots n$.

2. For $b=1 \ldots B$, do:

- Compute $z_{i j}=\left(x_{i j}-\bar{X}_{i}\right) / S_{i}, \forall i=1 \ldots n, j=1 \ldots J$.

- For each gene, obtain a sample of size $J$ with replacement from the collection of all $z_{i j}$. Denote this sampled values as $z_{i j}^{(b)}$. Then compute $x_{i j}^{(b)}=S_{i} z_{i j}^{(b)}+\bar{X}_{i}$.

- Apply the procedure to find differentially expressed genes to the bootstrap dataset. Since all discoveries are false positives, denote the number of genes declared $\mathrm{DE}$ as $\mathrm{FP}_{b}$.

3. Estimate the frequentist FDR as $\widehat{\mathrm{FDR}}=\hat{\pi}_{0} \frac{\sum_{b=1}^{B} \mathrm{FP}_{b}}{B \times P}$, where $\hat{\pi}_{0}$ is an estimate of the proportion of EE genes.

There is an important remark to make here: there are other ways to simulate data under the null hypothesis. For instance, one could simply permute the group labels or bootstrap data within each gene, but this could be troublesome when the sample size is too small to provide an accurate representation of the null. Determining what sample size is large enough is not a simple matter.

For example, suppose that we have a gene for which most expression values are small, but for one of the groups it occasionally presents large expression values. Table 110 presents hypothetical values for a single gene and 2 groups. Note that there are $\left(\begin{array}{c}10 \\ 5\end{array}\right)=184,756$ ways to permute the group labels, which at first sight may seem a large enough number. However, in group 2 there is an outlying value. For any permutation, whatever group the outlier is assigned to will tend to be declared to have higher expression levels 


\begin{tabular}{|c|c|}
\hline Group 1 & Group 2 \\
\hline 2.2 & 2.8 \\
2.8 & 3.2 \\
3.1 & 2.0 \\
2.5 & 2.1 \\
2.7 & 2.1 \\
2.0 & 2.5 \\
2.1 & 2.9 \\
2.3 & 10.5 \\
2.9 & 2.2 \\
2.4 & 2.4 \\
\hline
\end{tabular}

Table 1: Hypothetical expression values for a gene, 10 arrays and 2 groups

than the other group, and it will be counted as a false positive. If one obtains a bootstrap sample, there is some probability that the effect of the outlier will be mitigated (the outlier may not be sampled at all, or be sampled the same number of times in both groups), but it will still tend to increase to false positive count. If one bootstraps residuals from other genes, such as Algorithm 1 does, the value of 10.5 may not be outlying at all anymore, since other genes may present values that are also far away from the mean.

Hence, we see that three reasonable strategies to sample under the null may result in three quite different sampling distributions and estimated FDR. The main issue is whether the outlying value should be considered to be an error or not. In our experience, in microarrays it is not unfrequent to encounter data such as that in Table 1. A possible biological explanation is that a small proportion of individuals from group 2 experience some kind of mutation, which causes the expression of a particular gene to raise considerably. If such a discovery is biologically meaningful one should not count it as a false positive, and hence methods based on permuting or bootstrapping each gene separately would not be appropriate. We agree with (7) that more research is needed regarding this topic, but we feel that unless the number of arrays is quite large it may be beneficial to use a resampling scheme that uses data from several genes at once.

\subsection{Frequentist FDR for the Armstrong dataset}

We now estimate the frequentist FDR of the Bayesian procedure outlined in Section 2.1 by applying Algorithm 1 to the Armstrong dataset.

Figure 2(a) displays the estimated frequentist FDR for target Bayesian 
GaGa model

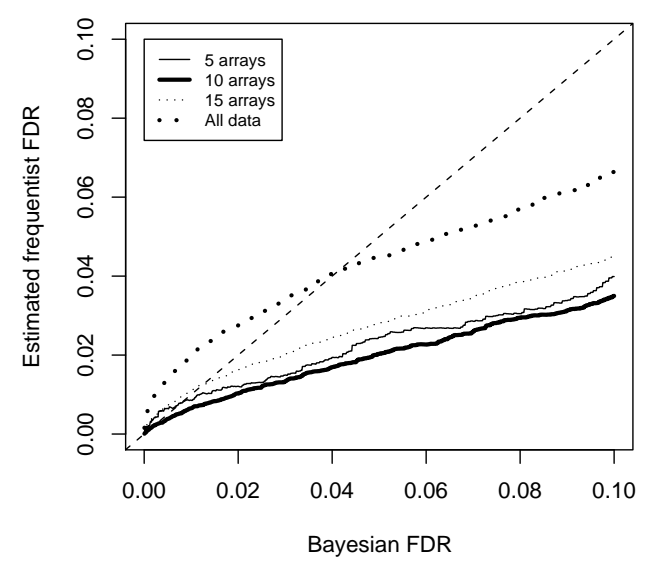

MiGaGa model $(M=2)$

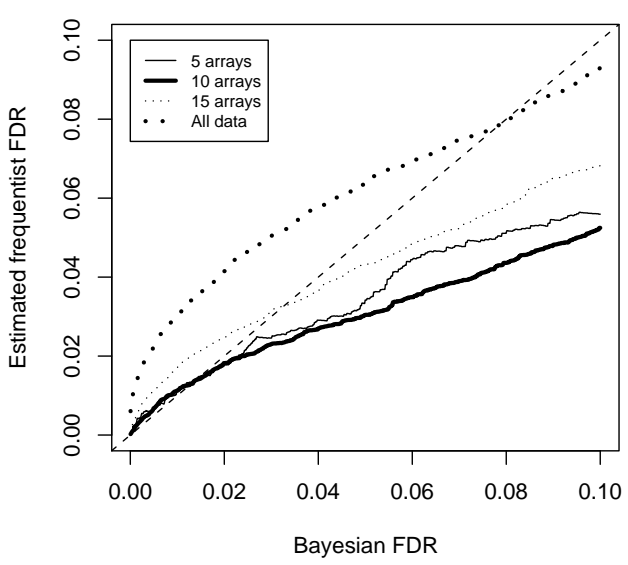

Figure 2: Armstrong dataset. Frequentist FDR for the GaGa (a) and MiGaGa (b) models.

FDRs ranging from 0 to 0.1 , both for the GaGa and MiGaGa models and increasing amounts of data. For Bayesian FDR at the 0.05 level, the estimated frequentist FDR is always below 0.05. The only exception is the MiGaGa model applied to the full dataset, for which the frequentist FDR is estimated to be $6.4 \%$.

Figure 3 provides the analogous estimates for the monotonically transformed data, which was analyzed with a GaGa model. For a Bayesian FDR of 0.05, the estimated frequentist FDR is below 0.05, as desired.

We conclude that the Bayesian procedure from Section 2.1 has reasonably good frequentist operating characteristics when applied to the Armstrong dataset.

\section{Goodness of fit in the Armstrong dataset}

In the original paper, Section 6.2.1, we evaluated some aspects of the overall goodness-of-fit. Figure 3(b) compares the marginal distribution of the monotonically transformed data with draws from the prior-predictive GaGa model, setting the hyper-parameters to their posterior mean. The monotonic transformation improves the fit of the GaGa model substantially (compare with Figure 4(a) in the original paper).

We now assess the fit for some genes individually. First, we select the two 
(a)

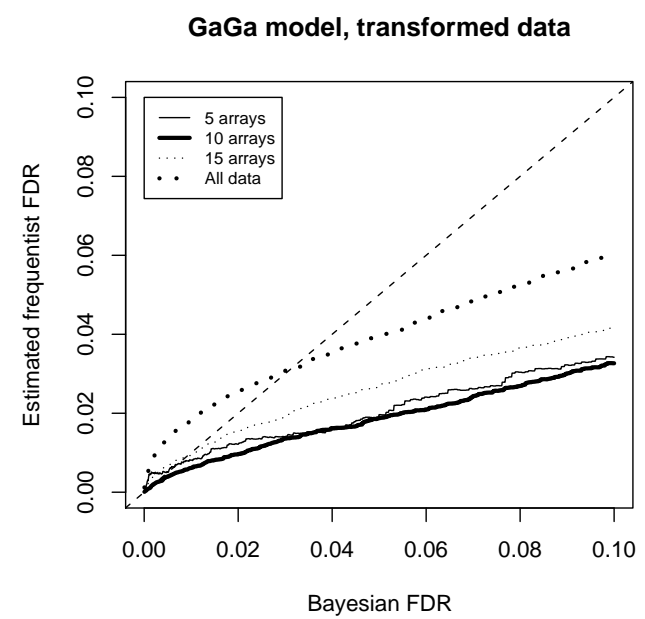

(b)

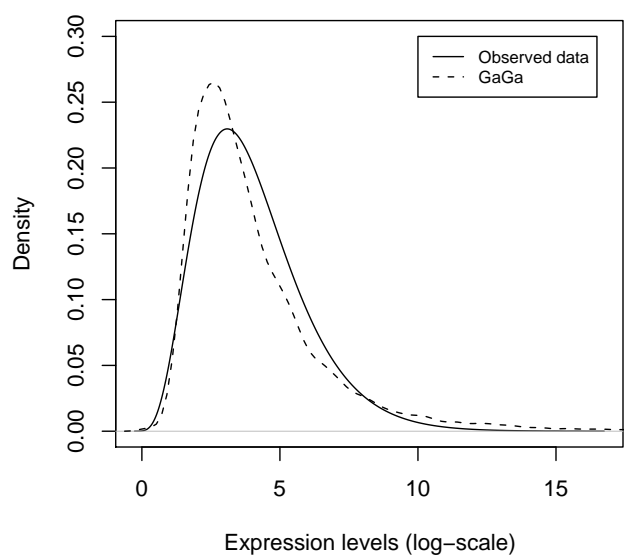

Figure 3: Armstrong dataset after monotonic transformation. (a): frequentist FDR fot the GaGa model; (b): marginal distribution of data vs. prior predictive of GaGa model with $\boldsymbol{\omega}=\hat{\boldsymbol{\omega}}$.

genes with the highest probability of being DE according to the Ga model. Figure 4(a) compares their observed expression values with draws from their posterior predictive distribution based on the Ga model. We see that, even though the model underestimates the variability for the MLL group, the two genes do actually seem to be differentially expressed. Figure 4(b) shows how draws from the GaGa model posterior predictive more appropriately capture the variability of the data. For these two genes in particular, however, the result of the inference is the same: both models declare probes 1914_at and 37809_at to be differentially expressed.

We now select two genes that are declared DE by the Ga model and EE by the GaGa model, and again compare the observed values to their posterior predictive distribution. Figure 4(c) reveals that the Ga model underestimates the variability in the data, while in panel $(\mathrm{d})$ we see that GaGa represents it more satisfactorily. In this case the inference about the probes 1369_s_at and 2087_s_at from both models is radically different, for the GaGa model assigns a posterior probability $<0.01$ that each gene is differentially expressed. The poor Ga fit to these two genes and the fact that no strong differences between groups are observed in Figure 4(c)-(d) suggest that the inference provided by the GaGa model is more realiable. Although not presented here, we observe a similar favorable behavior of the MiGaGa model with $M=2$ components. 
(a)

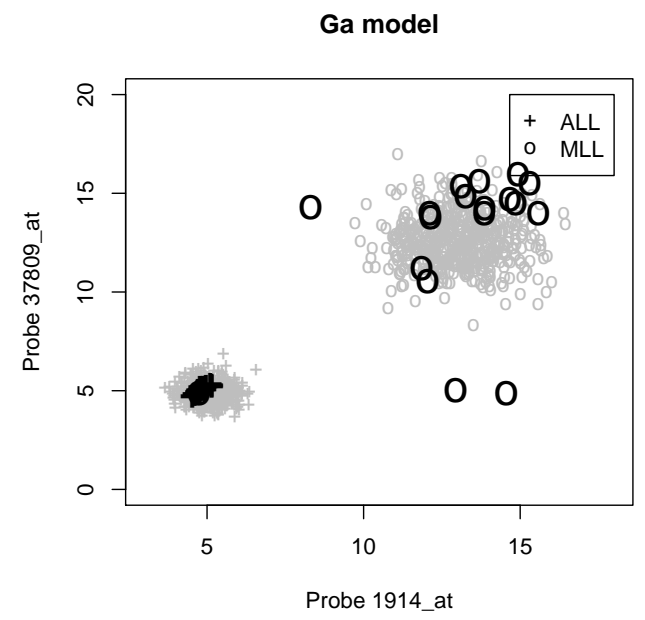

(c)

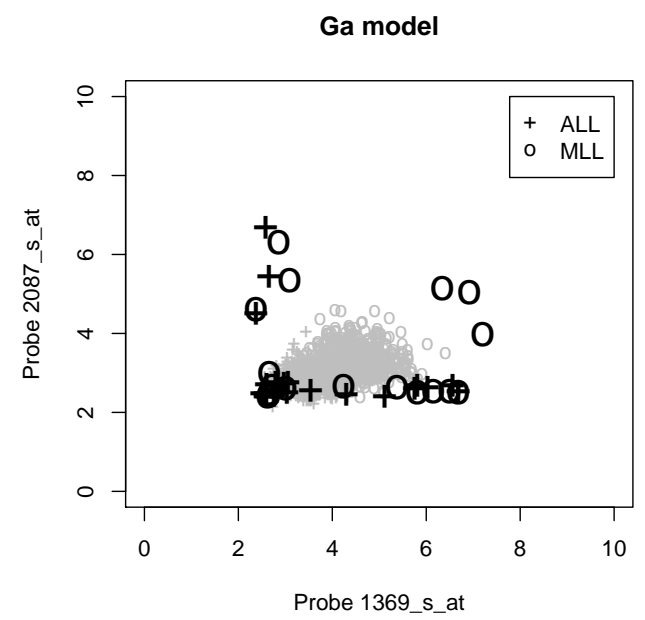

(b)

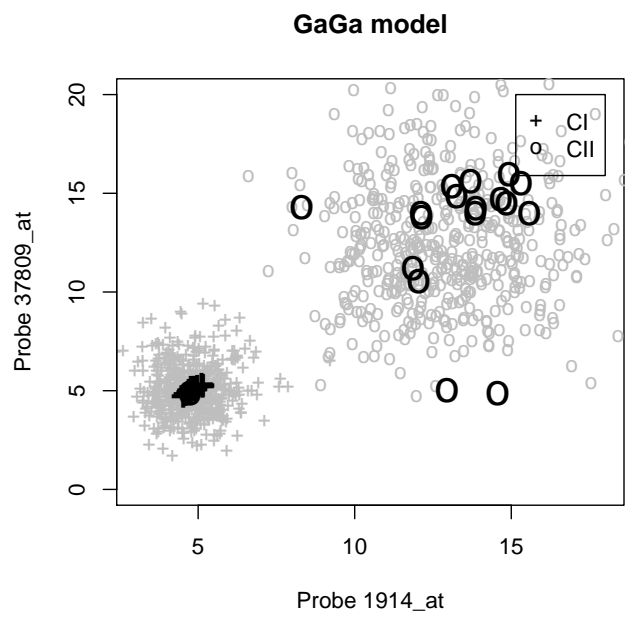

(d)

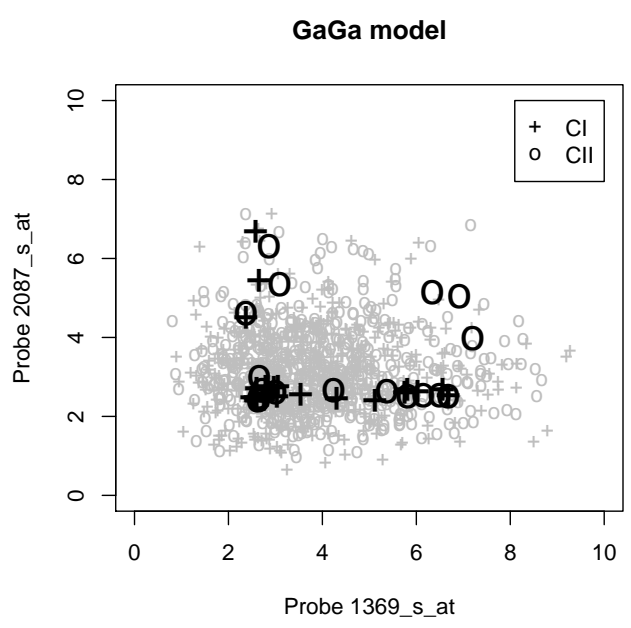

Figure 4: Observed expression values vs. predictive distribution. Large black symbols are actual observations, small gray symbols are draws from the posterior predictive. (a),(b): the two genes with highest probability of being DE according to the Ga model; (c),(d): two genes declared DE by the Ga model and declared EE by the GaGa model 
We conclude that the posterior distribution of the GaGa and MiGaGa models present an adequate fit to the data. This is in contrast with the prior-predictive plot in Figure 3(b) in the original paper, which suggested the GaGa fit to be of limited quality. This should not be too surprising, it merely reflects that the posterior distribution incorporates the information about bimodality present in the data.

\section{Gene ontology analysis for MAQC study}

The biological process gene ontology analysis was performed with the software DAVID (2). P-values for enrichment were computed with the hypergeometric test, and they were adjusted with the Benjamini-Hochberg procedure.

The complete listings of enriched categories can be found at http:// rosselldavid.googlepages.com. The file GO_gaga_BgtA.txt contains the results for the 1,000 genes with highest posterior probability of following Pattern 4, according to the GaGa model, and with the group B mean expression higher than that of group A. The file GO_gaga_AgtB.txt contains the same results for genes with group B mean lower than the group A mean. The files GO_migaga_BgtA .txt and GO_migaga_AgtB.txt contain analogous results for the MiGaGa model posterior probabilities.

\section{References}

[1] Armstrong, S., Staunton, J., Silverman, L., Pieters, R., Boer, M., Minden, M., Sallan, E., Lander, E., Golub, T., And KoRSMEYER, S. (2002). Mll translocations specify a distinct gene expression profile that distinguishes a unique leukemia. Nature Genetics 30, 41-47.

[2] Dennis, G., Sherman, B., Hosack, D., Yang, J., Baseler, M., LAne, H., AND Lempicki, R. (2003). David: Database for annotation, visualization, and integrated discovery. Genome Biology 4, (5):P3.

[3] Geman, D. and Geman, S. (1984). Stochastic relaxation, Gibbs distributions, and the Bayesian restoration of images. IEEE Transactions on Pattern Analysis and Machine Intelligence 6, 721-741.

[4] Hastings, W. (1970). Monte carlo sampling methods using markov chains and their applications. Biometrika 57, 97-109. 
[5] Müller, P., Parmigiani, G., and Rice, K. (2007). FDR and Bayesian Multiple Comparisons Rules. Oxford University Press.

[6] Müller, P., Parmigiani, G., Robert, C., and Rousseau, J. (2004). Optimal sample size for multiple testing: the case of gene expression microarrays. Journal of the American Statistical Association 99, 990-1001.

[7] Storey, J. (2007). The optimal discovery procedure: A new approach to simultaneous significance testing. Journal of the Royal Statistical Society B 69, 347-368. 